\title{
Transitivity of choice behavior in preschool children
}

JOSEPH C. CAMPIONE, ERIC G. GJESDAHL, KRISTEEN GRIFFIN, and F. MICHAEL RABINOWITZ, University of Washington, Seattle, Wash. 98105

An experiment was conducted demonstrating that experimentally-induced response strengths are transitive. Ss were trained to choose stimulus $A$ over stimulus $B$, stimulus $B$ over stimulus $C$, and stimulus $A$ over stimulus $C$. On test trials it was found that Ss more frequently chose $A$ over $C$ than either $A$ over $B$, or $B$ over $C$. These results are consistent with a large class of learning theories, and generalize the findings obtained in previous experiments to a wider class of subject and stimulus variables.

One prediction which is shared by many learning theories is that learned responses (choices) should be transitive. If a $S$ learns to choose Stimulus A over Stimulus B, and Stimulus B over Stimulus C, he should also come to choose Stimulus A over Stimulus C. Basically, this prediction comes about whenever a theorist assumes that there exists some scale (of habit strength, associative strength, response strength, etc.) which underlies choice behavior. If $\mathrm{S}$ must make a choice between two or more stimuli, he should tend to choose that stimulus which has the most, e.g., response strength. Theorists have differed in the way in which they have related response strengths to behavior. For example, Spence (1956) assumed that performance is determined by the difference between the strengths of the competing stimuli, Luce (1959) that performance is determined by the ratio between the strengths of the competing stimuli. When $\mathrm{S}$ prefers Stimulus A to Stimulus B, A must have more strength than B; similarly, if $S$ chooses B over $\mathrm{C}, \mathrm{B}$ must have more strength than C. From this it follows that A must have more strength than $\mathrm{C}$. Whether a difference or ratio rule is used, it also follows that A should be more strongly preferred to $C$ than both $A$ to $B$, and $B$ to $C$.

Despite the importance of this property, it has not been extensively investigated. In a recent series of experiments, Campione (1968) attempted to demonstrate that the experimentally-induced response strengths of retarded children were transitive. While the demonstration was successful, some difficulties encountered in the earlier experiments suggested that some boundaries exist on the conditions under which transitivity will hold. Further, some other questions dealing with the generality of the obtained results remained unanswered. The major aim of the present study was to attempt to generalize the results obtained in the previous experiments to normal children with a different class of stimuli.

In Campione's (1968) first experiment, use was made of junk pictures (pictures differing multidimensionally in color, form, size, texture, etc.). Briefly, Ss were given a long series of problems, each consisting of two training trials and one test trial. On one of the training trials, Stimuli A and B were presented, with $A$ being reinforced; on the other trial, Stimuli $B$ and $C$ were presented, with $B$ being reinforced. These training trials were followed by a test trial presenting either $A$ and $B, B$ and $C$, or A and C. Each S was tested 16 times on each of the three test trials. The experiment was unsuccessful in demonstrating transitivity, only two of 10 tests being consistent with the transitivity assumption. Two hypotheses were offered to account for this failure, the first suggesting that, for transitivity to be demonstrated, it is necessary that the stimuli used in any test vary along only one dimension. Accordingly, the second experiment (Experiment 1 in the report) was a replication of the first with the exception that form was the only dimension relevant to problem solution. Ss were pretrained prior to entry into the experiment to ensure the fact that they were attending to form and form alone. However, the second experiment also failed to demonstrate transitivity, leading to the final two experiments (Experiments 2 and 3 ).

The second hypothesis advanced to account for the failure of the first experiment dealt with the fact that the training-test relationship differed for the different test pairs. On the training trials, Stimuli A and B, and B and C were presented together. When these pairs were re-presented on the test trials, the pairings remained unchanged; however, when $A$ and $C$ were presented, a novel transverse pattern (Spence, 1952) was interjected. To the extent that Ss respond differentially to familiar and novel transverse patterns, this effect would invalidate any test of transitivity. Experiments 2 and 3 were designed to equate the test pairs with respect to novelty, Experiment 2 using a training procedure which made all test pairs novel, and Experiment 3 a training procedure which made all test pairs familiar. The stimuli used in both of these experiments again had form as the only relevant dimension. In both these experiments, transitivity was convincingly demonstrated, and it was found that Ss' performance on the A-C pair could be accurately predicted from knowledge of their performance on the A-B and B-C pairs. The predictions were derived from an equation taken from Luce (1959) and were based on the assumption that the scale underlying the response strength construct is a ratio scale. If we let $P(x, y)$ denote the probability that stimulus $x$ will be chosen when $x$ and $y$ are presented, and if we let $p_{1}=P(A, B)$ and $p_{2}=P(B, C)$, it can be shown that:

$$
P(A, C)=\frac{p_{1} p_{2}}{p_{1} p_{2}+\left(1-p_{1}\right)\left(1-p_{2}\right)}
$$

A chi-square goodness of fit test applied to the data of Experiments 2 and 3 led to the conclusion that the model could not be rejected.

While these experiments were interpreted as supporting the hypothesis that the earlier failures could be explained in terms of the argument regarding familiar and novel transverse patterns, the status of the hypothesis regarding stimulus redundancy remains in doubt. Merely using "unidimensional" stimuli was not sufficient to allow the demonstration of transitivity, but it still may be a necessary condition, since unidimensional stimuli were utilized in both successful experiments. The present experiment was carried out to see if redundant stimuli could be used in a successful demonstration of transitivity. Further, an attempt was made to generalize the findings to normal children.

\section{Subjects}

\section{METHOD}

Twelve children attending the preschool housed in the Developmental Psychology Laboratory at the University of Washington served as Ss. The Ss had a mean CA of 51 months (range: 44-60). Apparatus

A modified version of the Wisconsin General Test Apparatus was used. The $S$ and $E$ sat facing each other with a one-way vision screen interposed. The base of the apparatus contained a revolving circular tray ( 23 in. diameter) mounted on rollers. One half the tray contained two wells $5-1 / 4$ in. apart. Before each trial, E would place a marble in one of the wells, and put the stimuli into position. Then the tray would be revolved, presenting the stimuli to $S$.

The stimuli consisted of 132 junk pictures (pictures of everyday objects) mounted on 4 in. black cardboard squares.

Procedure

The procedure was similar to that used in Campione's (1968) Experiment 3 in which all test pairs consisted of familiar transverse patterns. Each experimental problem (EP) consisted of four trials: three training trials, and one test trial. The training trials consisted of one trial on which A and B were presented ( A reinforced), one trial on which $B$ and $C$ were presented ( $B$ reinforced), and one trial on which $A$ and $C$ were presented (A reinforced), followed by a test on one of the three pairs (nondifferential reinforcement was used on the test trial of any EP). There are six possible orders of the training trials and three possible test trials, making a total of 18 possible four-trial EP. Each EP was used twice, yielding a total of 36 problems per $S$, and allowing 12 tests per $S$ on each of the pairwise comparisons. Each $S$ was run for three days, a daily session consisting of one standard problem (SP-a series of four trials on which the same stimuli were 
Table 1

Proportions of Correct Responses on the Three Test Trials, and Predicted Proportions on the A-C Test. All Proportions are Based on an $\mathrm{N}$ of 12

\begin{tabular}{lccccc} 
Subject & A-B & B-C & A-C & A-C (Pred) & Outcome \\
\hline Danny & .33 & .42 & .33 & .27 & \\
*Stacey & .50 & .67 & .75 & .67 & + \\
*Maren & .67 & .75 & .83 & .86 & + \\
Jonathan & .42 & .58 & .58 & .50 & + \\
*Jill & .67 & .50 & .58 & .67 & + \\
Lara & .33 & .50 & .33 & .33 & + \\
*Peter & .67 & .83 & .92 & .91 & + \\
*Stephanie & .58 & .50 & .67 & .58 & + \\
Nancy & .42 & .33 & .25 & .27 & + \\
*Ben & .75 & .83 & .92 & .94 & .74 \\
*Dougie & .67 & .58 & .67 & .42 & + \\
Garth & .42 & .50 & .33 & & \\
\hline
\end{tabular}

*Transitivity test applicable

presented), $4 \mathrm{EP}, 1 \mathrm{SP}, 4 \mathrm{EP}, 1 \mathrm{SP}, 4 \mathrm{EP}$, and a final SP, for a total of 16 problems ( 64 trials) per day. For four-trial problems, there are a total of 16 right-left sequences which can be used. Eliminating those in which all four are rights or lefts (a total of two) and those in which the first three trials are rights or lefts (two) leaves a total of 12 position sequences. Throughout the course of the experiment, there were a total of $12 \mathrm{SP}$ and $36 \mathrm{EP}$, 12 each presenting either A-B, B-C, or A-C on the test trial (Trial $4)$, yielding four classes of problems. Within each class of problems, each of the 12 position sequences was used once. One further restriction on the position sequences throughout a day was that there were never more than three consecutive rights or lefts. The 44 stimuli to be used on a given day were selected randomly from the pool of 132, and the stimuli to be used on each problem were selected randomly without replacement from this set of 44 .

Each $S$ was brought individually to the experimental room where he was told he was going to play a game in which it was possible for him to win a prize if he won enough marbles. E then explained the rules of the game: $S$ was to look at the pictures that were presented and try to guess which one was right. He was to lift the card of his choice, finding a marble if he was correct (E would then say, "Yes, that's right."). If $S$ was wrong, there would be no marble, and E would say, "No, that's not right, it was under the other picture." A noncorrection procedure was used, with nondifferential reinforcement being given on the test trials of the EP.

\section{RESULTS AND DISCUSSION}

The results are summarized in Table 1 where it can be seen that the theoretical predictions were very accurate. A test for transitivity was made only if the estimates of $P(A, B)$ and $P(B, C)$ were both $\geqslant .5$. These conditions were met by seven of the $12 \mathrm{Ss}$, and in five of these cases, the transitivity assumption was borne out. Under the null hypothesis, transitivity should be found one-third of the time, and the probability of five or more successes out of seven tests is less than .05 (one-tailed test). Further, even when there was no test for transitivity, Equation 1 was still applicable to performance on the $\mathrm{A}-\mathrm{C}$ test, and there was close agreement between data and theory. For no $S$ was there a significant difference between obtained and predicted scores. As a final check on the applicability of the model to these data, a chi-square goodness of fit test was carried out, leading to the conclusion that the model could not be rejected $\left(x^{2}=3.25, \mathrm{df}=12, \mathrm{p}>.99\right)$. It should be emphasized that this test is at best approximate, given the facts that some of the expected frequencies were very small and that there was also error involved in the estimation of $P(A, B)$ and $P(B, C)$.

This experiment thus demonstrates that transitivity can be shown to hold even when highly redundant stimuli are used. These results are important for response strength theory, since they generalize the previous results to a different class of Ss (normal children, as compared to retarded children) and a different class of stimuli (multidimensional, as compared with unidimensional).

\section{REFERENCES}

CAMPIONE, J. C. Transitivity and choice behavior. Journal of Experimental Child Psychology, 1968, in press.

LUCE, R. D. Individual choice behavior. New York: Wiley, 1959.

SPENCE, K. W. The nature of the response in discrimination learning. Psychological Review, 1952, 59, 89-93.

SPENCE, K. W. Behavior theory and conditioning. New Haven: Yale University Press, 1956. 